\title{
Benign Oropharyngeal Neoplasm
}

National Cancer Institute

\section{Source}

National Cancer Institute. Benign Oropharyngeal Neoplasm. NCI Thesaurus. Code C4604.

A non-metastasizing neoplasm that arises from the oropharynx. 